\title{
Teil I: Beschlagnahmesicherheit und Forschungsgeheimnis
}

I.1. Grundsätzliches

I.2. Welche Rolle spielen arztrechtliche Belange beim Beschlagnahmeschutz?__ $\quad 147$

I.3. Welche Rolle spielt dabei die Rechtsform bzw. die Trägerschaft der BMB? ___ 147

I.4. Welche gesetzlichen Regelungen sind zur Etablierung eines Forschungsgeheimnisses für BMB erforderlich?__ 147

I.5. Welche strategischen und praktischen Konsequenzen hätte ein gesetzlich verankertes Forschungsgeheimnis für den Betrieb einer BMB?

I.6. Wie ist die intellektuelle Eigentümerschaft einer BMB geregelt und gesichert? 148 



\section{I.1. Grundsätzliches}

Proben und Forschungsdaten unterliegen dem Beschlagnahmeschutz im Ergebnis nicht.

Beschlagnahmevorschriften aus dem Zivilrecht ( $\iint 704 \mathrm{ff} Z \mathrm{ZPO}$ ) betreffen lediglich die Durchsetzung von Vermögensforderungen und sind daher nicht einschlägig. Verwaltungsrechtliche Vorschriften etwa nach BSeuchV, InfSG, UZwGBw etc. sind ebenfalls nicht passend. Ein Schutz vor Beschlagnahme wäre daher ausschließlich aus den Vorschriften der StPO ableitbar. ${ }^{200}$

Die Beschlagnahme ist vorrangig in den $\iint$ 94-IIIp StPO geregelt. Der \ 94 StPO erfasst alle körperlichen Gegenstände. Einer Beschlagnahme können daher Akten, Unterlagen sowie Datenträger und auch Proben unterliegen. Die Beschlagnahme von gespeicherten Daten darf sogar in Form einer Übermittlung auf den Computer der StA erfolgen. Ein Beschlagnahmeschutz existiert, wenn ein Gegenstand beschlagnahmefrei nach $\int 97$ StPO ist. \97 Abs. I Nr. I bis 3 StPO regelt die Beschlagnahmeverbote schriftlicher Mitteilungen (Nr. I), Aufzeichnungen (Nr. 2) und anderer Gegenstände einschließlich ärztlicher Untersuchungsbefunde ${ }^{201}$ und anatomischer Präparate ${ }^{202}$ (Nr. 3), die sich nach Abs. 2 im Gewahrsam der in $₫ 53$ Abs. I StPO genannten Personen befinden. ${ }^{203}$ Gemäß $\int 53$ Abs. I Nr. 3 StPO gehören dazu die zur Zeugnisverweigerung berechtigten RAe, Notare und Ärzte. Die Beschlagnahmesicherheit von Proben ist auch bei einem Rechtsanwalt gewährleistet, weil sich die Befreiung auf alles erstreckt, was ihm bei der Ausübung des Berufs anvertraut bzw. bekannt geworden ist. Zumindest muss die Erlangung des Wissens mit der Ausübung des Berufs zusammenhängen. Diese Kriterien sind im Falle von Proben erfüllt, so dass Anwälte zweifelsfrei mit unter die Befreiung fallen ${ }^{204}$. Eine Ausdehnung auf Angehörige anderer Berufe ist von Rspr. und Schrifttum durchweg abgelehnt worden. ${ }^{205}$ Treuhänder können sich demnach grundsätzlich nicht auf Beschlagnahmeverbote berufen. Auch das Zeugnisverweigerungsrecht der Berufshelfer nach $\int 53$ a StPO wird im Regelfall nicht einschlägig für eine Zuordnung zum geschützten Personenkreis sein. ${ }^{206}$

Der in $\int 53$ Abs. I Nr. 3 StPO benannte Personenkreis ist zur Verweigerung des Zeugnisses über dasjenige berechtigt, was ihm in dieser Eigenschaft anvertraut oder bekannt geworden ist. Nach diesem berufsbezogenen Zeugnisverweigerungsrecht sind also nur die in beruflicher Eigenschaft bekannt ge-

$200 \$ 46$ OwiG verweist auf die Vorschriften über das Strafverfahren, namentlich diejenigen der StPO.

201 Fraglich ist, ob Befunde aus genetischen Diagnosen ärztliche Untersuchungsbefunde oder innen gleichgestellt sind. Diese Frage ist in der juristischen Literatur bisher nicht beantwortet. Es dürfte aber wohl davon auszugehen sein, dass es sich um entsprechende Befunde handelt.

202 Meyer-Goßner, Lutz, $\emptyset 97$ StPO Rdnr. 30 m.w.Nw, von dem Begriff anatomische Präparate werden nach diesseitiger Ansicht auch Proben erfasst.

203 Zur Erläuterung der jeweiligen Begriffe in Nr. 1 bis 3 siehe etwa Pfeiffer, StP0, $\emptyset 97$ Rn. 2 ff.

204 Anderer Ansicht ist Goebel, Evaluation (2005), S. 27 f. mit dem Argument, es gehöre nach dem einschlägigen Berufsbild üblicherweise nicht zu den Aufgaben eines Anwalts, „Dinge für einen Mandanten aufzubewahren, die mit dem eigentlichen Beratungsfall nichts zu tun haben“. Der Beratungsfall kann sich hier aber gerade auf diesen Bereich erstrecken.

205 vgl. Löwe-Rosenberg-Dahs, StPO, \53 Rn. 4; dies. Schäfer, \97 Rn. 11; Meyer-Goßner, StPO, \97 Rn. 2.

206 vgl. so im Ergebnis Pfeiffer, StPO, $\oint 53$ a Rn. 1 f. 
wordenen Tatsachen oder erlangten Gegenstände geschützt. D.h. die Wissensoder Sachherrschaftserlangung muss unmittelbar aus der Berufsausübung herrühren. Es genügt allerdings die Kenntnis- oder Sachherrschaftserlangung aus Akten, Karteien etc. des Praxisvorgängers. Ein Praxisnachfolger kann daher ebenfalls Beschlagnahmeschutz verlangen. ${ }^{207}$

Das Zeugnisverweigerungsrecht der RAe und Notare betrifft alles, was sie bei der Berufsausübung erfahren. Der Begriff des Bekanntwerdens ist weit auszulegen. In Bezug auf Notare ist Beschlagnahmesicherheit bereits anzunehmen, wenn Unterlagen bei ihnen hinterlegt werden, denn die notarielle Hinterlegung ist eine typische Form der Berufsausübung der Notare. Das Zeugnisverweigerungsrecht der Ärzte erstreckt sich auf alles, was aus Anlass einer medizinischen Untersuchung oder Behandlung anvertraut oder bekannt geworden ist. ${ }^{208} \mathrm{Um}$ ein Zeugnisverweigerungsrecht und damit auch eine Beschlagnahmesicherheit der vom Arzt geführten Unterlagen auszulösen, bedarf es mithin eines Arzt-Patienten-Verhältnisses (vgl. etwa Meyer-Goßner, StPO, 『97 Rn. 4I). Das Beschlagnahmeverbot wirkt allerdings auch nach Beendigung der beruflichen Beziehung fort, soweit es sich um medizinische Daten handelt. ${ }^{209}$ Für Proben gilt dies entsprechend.

Gemäß $\int 97$ Abs. 2 StPO müssen sich die dem Beschlagnahmeschutz unterliegenden Gegenstände im Gewahrsam des geschützten Personenkreises befinden. Bei Ärzten genügt es gemäß Satz 2 auch, wenn die Gegenstände im Gewahrsam einer Krankenanstalt sind. Der Begriff der Krankenanstalten ist weit auszulegen. Er umfasst auch Genesungsheime, Pflegeanstalten und Krankenreviere der Bundeswehr, Polizei oder einer JVA. ${ }^{210}$ Allen genannten Einrichtungen ist gemein, dass dort Leiden behandelt werden. Reine Forschungsinstitute oder selbständige An-Institute dürften damit nicht gemeint sein. Eine Grauzone könnte sich an Universitätskliniken auftun, falls dort auf den normalen Krankenstationen am Patienten geforscht wird, z.B. durch nicht mit der Behandlung befasste Mediziner, und die für die Forschung gewonnen Proben und Daten von den Behandlungsunterlagen separiert werden. Auch bei den rechtsberatenden Berufen reicht Mitgewahrsam. ${ }^{211}$ Dies führen Löwe-Rosenberg-Schäfer mit Hinweisen zu den Gemeinschaftspraxen und die dortigen Gewahrsamslagen aus. ${ }^{212}$ Nur Lemke ${ }^{213}$ genügt Mitgewahrsam nicht.

In Grenzen liefert auch abgeleiteter Gewahrsam ein Beschlagnahmeverbot. ${ }^{214}$ Wenn der Gewahrsam von einem nach $\llbracket 53$ Abs. I Nr. 3 StPO geschützten Rechtsinhaber auf einen gleichfalls diesem Personenkreis Angehörigen übertragen wird, gilt der Beschlagnahmeschutz auch an der neuen Stelle..215

207 BVerfGE 32, 373 = NJW 1972, 1124; Pfeiffer, a.a.0., $\varnothing 97$ Rn. 6.

208 Also nicht auf reine Forschungsdaten.

209 vgl. Lemke/Julius/Krehl pp., StPO, $\oint 97$ Rn. 14; und im Ergebnis so wohl auch Meyer-Goßner, a.a.0., \53 Rn. 18.

210 vgl. Meyer-Goßner, a.a.0., \$ 97 Rn. 14.

211 vgl. etwa Meyer-Goßner, a.a.0., $\$ 97$ Rn. 12 und 40.

212 vgl. a.a.0., $\$ 97$ Rn. 29 m.w.N.

213 vgl. a.a.0., $\$ 97$ Rn. 9.

214 Löwe-Rosenberg-Schäfer, a.a.0., $\$ 97$ Rn. 9.

215 vgl. Meyer-Goßner, a.a.0., $\$ 97$ Rn. 11 und 13; Pfeiffer, a.a.0., $\oint 97$ Rn. 6; und zum Berufshelfer nach $\$ 53$ a StPO siehe ders., $\$ 53$ a Rn. 1 f. 
I.4. Welche gesetzlichen Regelungen sind zur Etablierung eines Forschungsgeheimnisses für BMB erforderlich?

Datenschutzbestimmungen stehen nach einschlägiger Kommentierung einer Beschlagnahme regelmäßig nicht entgegen, weil dem Schutz personenbezogener Daten auch Rechnung getragen wird, wenn sich die Unterlagen bei den Gerichtsakten befinden. Etwas entschärft wird die Möglichkeit der Einsichtnahme in die Unterlagen auch dadurch, dass letztere gemäß 『 IIO StPO nur durch den Staatsanwalt erfolgen darf. Dieser Aspekt ist allerdings nur für die erhobenen Daten, nicht aber für Proben relevant, da Proben als solche nicht dem Datenschutz unterliegen.

\section{I.2. Welche Rolle spielen arztrechtliche Belange beim Beschlagnahmeschutz?}

\203 Abs. I Nr. I Abs. 3 StGB stellt die unbefugte Offenbarung von Privatgeheimnissen durch Ärzte und ihr medizinisches Hilfspersonal unter Strafe. Sofern dieser Personenkreis Proben gewinnt und die Proben auch bei ihnen verbleiben, gilt der Beschlagnahmeschutz unabhängig von der Zweckbestimmung.

\section{I.3. Welche Rolle spielt dabei die Rechtsform bzw. die Trägerschaft der BMB?}

Zivilrechtlich spielt die Rechtsform keine Rolle. Strafbar können sich nur die natürlichen Personen machen, die einen Straftatbestand verwirklichen. Bei Gesellschaften können dies die Mitarbeiter und die Leitungsorgane sein ( $\mathbb{\text { I } 4}$ StGB). Vor allem letztere können sich auch durch Anstiftung, Beihilfe oder Begünstigung strafbar machen.

\section{I.4. Welche gesetzlichen Regelungen sind zur Etablierung eines Forschungsgeheimnisses für BMB erforderlich?}

Für die Etablierung eines Forschungsgeheimnisses wäre eine Ausweitung des Rechts auf Zeugnisverweigerung ( $\mathbb{5} 53$ Abs. I Satz I Nr. I bis 3 b StPO) auf medizinisches und humanbiologisches Forschungspersonal und des Beschlagnahmeverbots ( $\mathbb{9} 97$ Abs. I Nr. 3 StPO) auf die in einer BMB gewonnenen Daten und Proben erforderlich. In Ihrer Entschließung vom 25./26.3.2004 fordern die Datenschutzbeauftragten des Bundes und der Länder den Bundesgesetzgeber auf,

- „in $₫ 203$ StGB die unbefugte Offenbarung von personenbezogenen medizinischen Forschungsdaten unter Strafe zu stellen,

- in $\llbracket 53,53 \mathrm{a}$ StPO für personenbezogene medizinische Daten ein Zeugnisverweigerungsrecht für Forscher und ihre Berufshelfer zu schaffen, 
Teil I: Beschlagnahmesicherheit und Forschungsgeheimnis

- in $\int 97$ StPO ein Verbot der Beschlagnahme personenbezogener medizinischer Forschungsdaten zu schaffen.“" ${ }^{116}$

\section{I.5. Welche strategischen und praktischen Konsequenzen hätte ein gesetzlich verankertes Forschungsgeheimnis für den Betrieb einer BMB?}

Bei einem gesetzlich verankerten Forschungsgeheimnis wären die in BMB gewonnenen Daten und Proben vor dem Zugriff von Polizei und Staatsanwaltschaft geschützt. Proben, Daten und Datenträger dürften im Wege der Beschlagnahme nicht gegen den Willen der Betreiber und der unmittelbar beteiligten Forscher aus deren Gewahrsam entfernt und mitgenommen werden. Rechtspraktisch wäre dies ein großer Gewinn. Insbesondere könnte dadurch die Bereitschaft der Spender wachsen, ihre Proben und Daten einer BMB bzw. der Forschung anzuvertrauen. Es bleibt allerdings zu bedenken, dass durch die Etablierung eines Forschungsgeheimnisses eine Art von rechtsfreiem Raum entstehen könnte. Wenn Ermittlungsorgane auf verbotswidrig gesammelte Proben und Daten nicht zugreifen dürfen, entgeht ihnen Beweismaterial, mit dem strafbares Verhalten in der Forschung möglicherweise erst verfolgt werden könnte. Mit Goebel ${ }^{217}$ ist darauf hinzuweisen,

„dass ein umfassendes Forschungsgeheimnis nicht nur die strafprozessuale, sondern auch die Seite der Gefahrenabwehr durch die Organe der Vollzugspolizei sowie der Sonderpolizeibehörden (des Zolls usw.) erfassen müsste. Dies gilt insbesondere, nachdem der Gesetzgeber aktuell unter der Fahne der ,Bekämpfung des internationalen Terrorismus' allenthalben präventiv-polizeiliche Eingriffsstrukturen einführt oder dies abzusehen ist“.

\section{I.6. Wie ist die intellektuelle Eigentümerschaft einer BMB geregelt und gesichert?}

Zweck einer BMB ist die Sammlung, Verarbeitung und Weitergabe von Proben und der damit verbundenen Daten. Demzufolge handelt es sich bei einer BMB um eine Proben- und Datenbank, sofern die Daten entsprechend systematisch verarbeitet werden. Ein Schutzbedürfnis könnte deshalb sowohl für die gespeicherten Proben und Daten als auch für die geistige bzw. intellektuelle Leistung bestehen die mit der Verarbeitung der Proben und Daten verbunden ist. 
Von „intellectual property rights“ spricht man im angelsächsischen Sprachraum, von „geistigem Eigentum“ im deutschen Sprach- bzw. Rechtsraum. Im „Agreement on trade-related aspects of intellectual property rights” vom I5.4.I994 (TRIPS), das auch für Deutschland gilt, umfasst der Begriff des geistigen Eigentums nach Art. I Abs. 2 das Urheberrecht und verwandte Schutzrechte, Patente sowie den Schutz nicht offenbarter Informationen. ${ }^{218}$ Von geistigem Eigentum wird in Deutschland insbesondere in der verfassungsrechtlichen Literatur gesprochen. ${ }^{219}$ In der bayerischen Verfassung werden das geistige Eigentum, das Recht der Urheber, Erfinder und Künstler gemäß Art. I62 ausdrücklich unter den Schutz des Staates gestellt. ${ }^{220}$ Die konkreten, verkörperten Rechte werden je nach ihrer Eigenart mehr oder weniger und in unterschiedlicher Art geschützt. Im Falle der BMB kommt in erster Linie der Schutz der Proben- und Datensammlung in Frage und weniger der Schutz einer sonstigen intellektuellen Urheberschaft. Dies liegt an der Zielrichtung der BMB, Proben zu erheben, zu verarbeiten und weiterzuleiten, nicht aber, sie selbst zu Zwecken der wissenschaftlichen Forschung zu benutzen.

\section{I.6.1. Schutz von Proben}

Ein Urheberschutz nach dem Urhebergesetz ist für die Probensammlung nicht gegeben, weil es sich dabei in der Regel nicht um eine schützenswerte geistige Leistung handelt ( $\mathbb{4} 4$ Abs.I Urhebergesetz). Sollte dies doch der Fall sein, ist unter Umständen ein Schutz gegeben. ${ }^{221}$ Davon zu unterscheiden ist die in den Proben verkörperte wirtschaftlich-organisatorische Leistung, die für solche Betriebsmittel als Recht am Unternehmen bzw. Gewerbebetrieb durch richterrechtliche Schöpfung geschützt wird.222 Dieses Recht hat die Funktion, den Schutz gegen Beeinträchtigungen der wirtschaftlichen Betätigung über das Wettbewerbsrecht und die deliktischen Normen der $\iint 823 \mathrm{ff}$. BGB hinaus zu gewährleisten, weil der Unternehmer vielfach ansonsten gegenüber fahrlässigen Eingriffen schutzlos wäre. ${ }^{223}$ Es umfasst Betriebsräume- und -grundstücke, aber auch Betriebsgeheimnisse. Voraussetzungen für die Gültigkeit sind Selbständigkeit, Entgeltlichkeit und Nachhaltigkeit des Geschäftsbetriebs sowie ein Auftreten nach außen. Ob und inwieweit eine BMB diese Kriterien erfüllt, wäre im Einzelnen zu prüfen.

\section{I.6.2. Schutz des Datenbankherstellers}

Einen spezifischen Schutz sieht das Urhebergesetz in $\int 87$ a für Datenbanken vor. Es folgt hier den Vorgaben der EG-Datenschutzrichtlinie, die der deutsche

218 Münchener Anwaltshandbuch (2005), Gewerblicher Rechtsschutz (Hrsg. G. Hasselblatt), 2. Aufl. München 2005, $\$ 1$ Rdnr. 23.

219 Vgl. BVerfG 31, 255.

220 Rehbinder (1998), Urheberrecht, 10. Aufl. München, S. 71.

221 Siehe dazu im Einzelnen unten.

222 H. Schack (1997), Urheber- und Urhebervertragsrecht, Tübingen, S. 119 Rdnr. 259.

223 Staudingers Kommentar zum Bürgerlichen Gesetzbuch, Zweites Buch, Recht der Schuldverhältnisse, 13. Bearbeit. V. J. Hager, Berlin 1999, \823 D 2. 
Gesetzgeber zum I.I.I998 umgesetzt hat. Ziel der Vorschrift ist es, den Datenbankhersteller in seinem Amortisationsinteresse vor der „parasitären“ Übernahme wesentlicher Teile seiner Datenbank zu schützen, ${ }^{224}$ indem ihm nach 『 $87 \mathrm{~b}$ das ausschließliche Recht gewährt wird, die Datenbank insgesamt oder in nach Art und Umfang wesentlichen Teilen zu vervielfältigen, zu verbreiten und öffentlich wiederzugeben. Voraussetzung für die Gewährleistung des Schutzes ist nach $\int 87 a$ UrhG, dass Daten

„systematisch oder methodisch angeordnet und einzeln mit Hilfe elektronischer Mittel oder auf andere Weise zugänglich sind und deren Beschaffung, Überprüfung oder Darstellung eine nach Art oder Umfang wesentliche Investition erfordert".

Geschützt werden allerdings nur Datenbanken

„bei denen entweder die Beschaffung, die Überprüfung, die Darstellung oder eine Änderung der Inhalte eine nach Art oder Umfang wesentliche Investition erfordert",

wobei der Begriff der wesentlichen Investition weder im Gesetz, der Datenbankrichtlinie noch in den dort vorangestellten Erwägungsgründen des Gesetzgebers näher erläutert wird. In Erwägungsgrund 40 wird lediglich festgestellt, dass finanzielle Mittel, Zeit, Arbeit oder Energie, unter Umständen (nach Erwägungsgrund 55) sogar eine intensive Überprüfung des Inhalts einer Datenbank eine wesentliche Investition sein können. Die Investition kann dabei in der Beschaffung von Informationen,

„in der Überprüfung der Datenbankelemente auf Vollständigkeit, Richtigkeit und Aktualität oder in der Aufbereitung für die Weitergabe durch die Erstellung von Abstracts, Tabellen, Verknüpfungen, Abfrageoptionen, Thesauri oder der Gliederung des Datenmaterials und seiner Indexierung liegen “.225

Es reicht aber aus, wenn sich die Investition von Geld, Zeit oder Arbeit lediglich auf einen inhaltlichen Teil der Datenbank erstreckt. Die Investition ist dann wesentlich, wenn sie substantielles Gewicht hat.

Bei der Gewinnung der Proben einer BMB, ihrer Verarbeitung und ihrer Weiterleitung werden Datenbanken erstellt, die die Kriterien der nach \87a UrhG erforderlichen Investition des Datenbankherstellers erfüllen. Somit ergibt sich der nach $\int 87 \mathrm{~b}$ UrhG erforderliche Schutz. Sollte allerdings lediglich eine einfache Sammlung von Daten vorliegen wie z.B. Namenslisten der Spender mit der Nummer der dazu gehörigen Probe, so sind die Kriterien für das Vorhandensein einer Datenbank unter Umständen nicht erfüllt. Dies muss im Einzelfall geprüft werden. 


\section{I.6.3. Intellektuelle Eigentümerschaft von Forschungsergebnissen}

Wenn sich die Aufgaben einer BMB lediglich auf das Erheben, Verarbeiten und Weiterleiten von Proben und Daten beziehen, sind weitere Schutzmöglichkeiten ausgeschlossen. Forschungsergebnisse, die von der BMB an Proben erzielt werden, könnten hingegen unter bestimmten Voraussetzungen über Patente oder nach \I UrhG in Form der Urheberschaft von Werken der Wissenschaft geschützt werden. Wenn es sich bei den Ergebnissen um eine „persönliche geistige Schöpfung " handelt, die durch Darstellungen wissenschaftlicher oder technischer Art, wie Zeichnungen, Plänen, Karten, Skizzen, Tabellen oder plastischen Darstellungen eine wahrnehmbare Form gefunden hat, Individualität ausweist und die notwendige Gestaltungshöhe erreicht hat, käme möglicherweise ein Schutz nach \2 Abs. I Nr. 7 UrhG in Frage. Handelt es sich um sprachliche Darstellungen, also wissenschaftliche Schriften, sollten sie durch $₫ 2$ Abs. I Nr. I UrhG geschützt sein.

\subsubsection{Zusammenfassung}

Die „intellektuelle Eigentümerschaft“ an der Erhebung, Verarbeitung und Weiterleitung von Proben ist nicht spezifisch geschützt. Wird im Zusammenhang damit eine Datenbank aufgebaut, gibt es für sie einen Schutz nach $₫ 87 \mathrm{a}$ und $\mathrm{b}$ UrhG. Unabhängig davon können Forschungsergebnisse bei einer bestimmten Erfindungshöhe als Patente geschützt werden, die sprachlichen oder sonstigen Darstellungen wissenschaftlicher oder technischer Art nach \2 UrhG. 